\title{
Qualität in der Medizin: Was (potentiell) messbar ist*
}

\section{Hans Stalder}

Prof. Dr. med., Mitglied der Redaktion

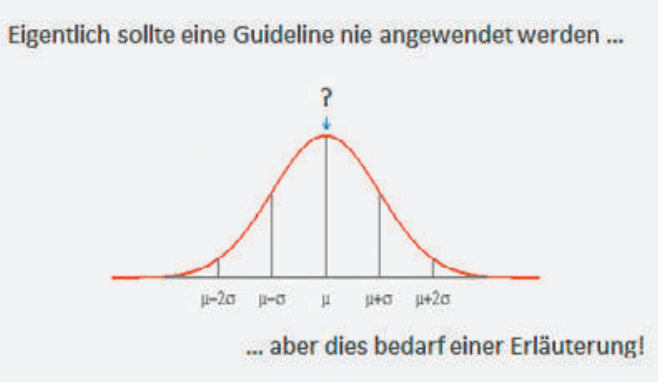

Nutzen Sie die Webseite der Schweizerischen Ärztezeitung? Wenn ja, dann finden Sie unter dem Stichwort "Qualität» über 2500 Treffer. Das heisst, die Mediziner kümmern sich um Qualität! Ein grosser Teil dieser Artikel beschäftigt sich mit Empfehlungen für die klinische Praxis, häufiger mit dem englischen Begriff Guidelines bezeichnet. Ohne auf bereits Gesagtes einzugehen, möchte ich im Zusammenhang mit Guidelines zwei Aspekte erörtern: Zunächst die Frage, wer ist wirklich qualifiziert, sie herauszugeben? Ein Blick auf die Mammographie soll das deutlich machen: In den USA empfehlen die Task Force für Präventivmedizin (USPSTF) und die Hausärzte bei den 50- bis 74-Jährigen eine Mammographie alle zwei Jahre, die Onkologen und der Ärzteverband sprechen sich für eine jährliche Untersuchung ab 40 aus und die Gynäkologen/Geburtshelfer wollen Frauen ab einem Alter zwischen 40 und 49 alle ein bis zwei Jahre untersuchen, ab 50 jährlich [1]. Solche Divergenzen finden sich in vielen anderen Bereichen und auch bei uns. Wie kommen Guideline-Autoren, die sich doch auf dieselben wissenschaftlichen Erkenntnisse stützen, zu so unterschiedlichen Empfehlungen? Die Vermutung liegt nahe, dass die Interpretation der statistischen Daten von standespolitischen und finanziellen Interessen beeinflusst ist. Die Empfehlung des Swiss Medical Board (SMB), auf ein Mammographie-Screening zu verzichten, hat nicht nur Proteste von Fachgesellschaften bewirkt (was nicht erstaunt), sondern auch Kritik ideologischer Gruppen und sogar von Politikern ausgelöst. So vermutete der Genfer Gesundheitsminister gar eine Zürcher Verschwörung gegen die offenbar solidarischeren Gesundheitssysteme der Westschweiz! Es gilt also, aufmerksam zu beobachten, wer als Autor hinter Guidelines steckt. Häufig verbergen sich Motive dahinter, die zu einer Verzerrung wissenschaftlicher Daten führen. Wer also sollte die Guidelines verfassen? Das genannte Beispiel macht deutlich, dass Ärzte allein, bzw. Fachleute nicht vor Verdacht gefeit sind. Der Staat? Der Vor- schlag des Bundesrats zur Einrichtung eines Qualitätszentrums hat es nicht einmal durch die Beratungen geschafft. Das SMB? Ja, vorausgesetzt es agiert vollständig unabhängig. Sollte sich herausstellen, dass die Industrie tatsächlich - wie heute diskutiert - einen Teil seiner Finanzierung übernehmen will, können seine Schlussfolgerungen nicht mehr ernst genommen werden. Also warum nicht die Schweizerische Akademie der Medizinischen Wissenschaften?

Ein weiteres Problem im Zusammenhang mit Guidelines ist ihre Umsetzung. Die jüngsten Ergebnisse einer Studie über die Wirkung zweier Guidelines des SMB sind nicht gerade ermutigend, denn sie zeigen, dass die Wirkung praktisch gleich null ist [2]. Wie also umsetzen? Sind Ärzte, die den Guidelines nicht folgen, zu sanktionieren, wie es das KVG, Art. 57 vorsieht? «Leistungserbringer, die den Vorgaben zur Wirtschaftlichkeit und zur Qualitätsgarantie nicht folgen [...] werden sanktioniert.» Wäre es nicht intelligenter, jene zu belohnen, die sie befolgen, wie es die Briten mit ihrem pay-for-performance system (P4P) machen? Wenn der Allgemeinmediziner in Grossbritannien auf 146 Performanceindikatoren, von denen $70 \%$ klinisch sind, die maximale Punktzahl von 1050 umsetzt, kann er sein Einkommen bis zu $25 \%$ steigern! Dieses System wurde relativ gut aufgenommen und die Ergebnisse lagen klar über den Erwartungen. Allerdings hat es auch seine Tücken: Die Ärzte könnten versucht sein, Patienten, die wenig geneigt sind, den Empfehlungen zu folgen, nicht anzunehmen und sich vorzugsweise mit jenen Kriterien zu befassen, die Punkte bringen - zu Lasten der anderen. Und schliesslich weiss man nicht, ob es den Patienten wirklich besser geht [3]. Wir brauchen uns jedoch keine Sorgen zu machen, denn dieses äusserst bürokratische System wird mangels einheitlicher elektronischer Daten in der Schweiz ohnehin nicht funktionieren.

Schliesslich sollten Guidelines niemals einfach tel quel befolgt werden. Die Patientengruppe, auf die sie sich stützen, entspricht nie genau unserem Patienten, und selbst wenn dies so wäre, würde er nie genau dem Durchschnitt der Gruppe entsprechen. Aber man müsste sich jedes Mal rechtfertigen, wenn man eine Guideline nicht umsetzt. Die Qualität dieser Rechtfertigung zu kontrollieren, erscheint mir eher schwierig. 